\title{
FOUNDATIONS OF ORDERED (SEMI)HYPERRINGS
}

\author{
SABer OMidi AND BiJan Davvaz \\ Department of Mathematics, Yazd University, Yazd, Iran \\ omidi.saber@yahoo.com,davvaz@yazd.ac.ir
}

\begin{abstract}
In the present paper, we introduce the notion of (semi)hyperring $(R,+, \cdot)$ together with a suitable partial order $\leq$. This structure is called an ordered (semi)hyperring. Also, we present several examples of ordered (semi)hyperrings and prove some results in this respect. By using the notion of pseudo order on an ordered (semi)hyperring $(R,+, \cdot, \leq)$, we obtain an ordered (semi)ring. Finally, we study some properties of pseudoorder on an ordered (semi)hyperring.
\end{abstract}

Key words and Phrases: Algebraic hyperstructure, ordered (semi)hyperring, strongly regular relation, pseudoorder.

\begin{abstract}
Abstrak. Dalam makalah ini diperkenalkan notasi dari (semi)hyperring $(R,+, \cdot)$ bersama dengan suatu suitable partial order $\leq$. Struktur ini disebut ordered (semi)hyperring. Beberapa contoh dari ordered (semi)hyperring disajikan dan dibuktikan. Dengan menggunakan notasi dari pseudoorder pad suatu ordered (semi)hyperring $(R,+, \cdot, \leq)$, diperoleh suatu ordered (semi)ring. Beberapa sifat dari pseudoorder pad suatu ordered (semi)hyperring.

Kata kunci: Algebraic hyperstructure, ordered (semi)hyperring, strongly regular relation, pseudoorder.
\end{abstract}

\section{INTRODUCTION}

Similar to hypergroups, the hyperrings extend the classical notion of rings. There exist different types of hyperrings. The more general structure that satisfies the ring-like axioms is the hyperring in the general sense. De Salvo [11] studied hyperrings in which the additions and the multiplications were hyperoperations; also see $[1,9,23,27,30]$. We refer the reader to Davvaz and Leoreanu-Fotea [8] for basic notions updated to 2007 of hyperring theory. Algebraic hyperstructures are a suitable extension of classical algebraic structures. In a classical algebraic structure, the composition of two elements is an element, while in an algebraic

2000 Mathematics Subject Classification: 16 Y99.

Received: 23-12-2015, revised: 04-08-2016, accepted: 24-08-2016. 
hyperstructure, the composition of two elements is a set. In 1934, the concept of hyperstructure was first introduced by a French mathematician, Marty [20], at the eighth Congress of Scandinavian Mathematicians where he defined hypergroups as a generalization of groups. Several books have been written on this topic, for example, see $[4,5,8,29]$. Further introduction to semihypergroups and hypergroups can be found in [4]. In [28], Vougiouklis introduced the concept of semihyperring, where both the addition and multiplication are hyperoperation. Semihyperrings extend the classical notion of semirings. There exist different types of semihyperrings. The more general structure that satisfies the semiring-like axioms is the semihyperring in the general sense.

Bakhshi and Borzooei [2] introduced the notion of ordered polygroups. Heidari and Davvaz introduced and studied ordered semihypergroup in [15]. Chvalina [6] and Hort [16] used ordered structures for the construction of hypergroups. Chvalina [6] started the concept of ordered semihypergroups as a special class of hypergroups in 1994. The concept of ordered semihypergroups is a generalization of the concept of ordered semigroups. Several authors have recently studied different aspects of ordered semihypergroups, for instance, Changphas and Davvaz [3], Chvalina and Moucka [7], Davvaz et al. [10], Gu and Tang [14], Heidari and Davvaz [15], Hoskova [17], Tang et al. [25], and many others. By an ordered semihypergroup, we mean an algebraic hyperstructure $(S, \circ, \leq)$, which satisfies the following conditions: (1) $(S, \circ)$ is a semihypergroup together with a partial order $\leq$; (2) If $x, y$ and $z$ are elements of $S$ such that $x \leq y$, then $z \circ x \leq z \circ y$ and $x \circ z \leq y \circ z$. Here, $z \circ x \leq z \circ y$ means for any $a \in z \circ x$ there exists $b \in z \circ y$ such that $a \leq b$. The case $x \circ z \leq y \circ z$ is defined similarly.

In this paper, we define the notion of ordered (semi)hyperrings and give some examples. Moreover, we define pseudoorder on ordered (semi)hyperrings and also a connection between ordered (semi)hyperrings and ordered (semi)rings has been investigated.

\section{BASIC DEFINITIONS AND PRELIMINARIES}

In this section, we recall some definitions and notations that will be used in the sequel.

A mapping $\circ: S \times S \rightarrow \mathcal{P}^{*}(S)$, where $\mathcal{P}^{*}(S)$ denotes the family of all nonempty subsets of $S$, is called a hyperoperation on $S$. The couple $(S, \circ)$ is called a hypergroupoid. In the above definition, if $A$ and $B$ are two non-empty subsets of $S$ and $x \in S$, then we denote

$$
A \circ B=\bigcup_{\substack{a \in A \\ b \in B}} a \circ b, A \circ x=A \circ\{x\} \text { and } x \circ B=\{x\} \circ B \text {. }
$$

A hypergroupoid $(S, \circ)$ is called a semihypergroup if for all $x, y, z \in S, x \circ(y \circ z)=$ $(x \circ y) \circ z$, which means that

$$
\bigcup_{u \in y \circ z} x \circ u=\bigcup_{v \in x \circ y} v \circ z .
$$


A hypergroupoid $(S, \circ)$ is called a quasihypergroup if for all $x \in S$, we have $S \circ x=x \circ S=S$. This condition is also called the reproduction axiom. A hypergroupoid $(S, \circ)$ which is both a semihypergroup and a quasihypergroup is called a hypergroup.

A comprehensive review of hyperrings theory is covered in Davvaz and Vougiouklis [9], Spartalis [24], Vougiouklis [30] and in the book [8] written by Davvaz and Leoreanu-Fotea. In the following, we consider one of the most general types of hyperrings.

Definition 2.1. A triple $(R,+, \cdot)$ is a (general) hyperring, if:

(1) $(R,+)$ is a hypergroup;

(2) $(R, \cdot)$ is a semihypergroup;

(3) The hyperoperation - is distributive with respect to the hyperoperation + , i.e., for all $a, b, c \in R, a \cdot(b+c)=a \cdot b+a \cdot c$ and $(a+b) \cdot c=a \cdot c+b \cdot c$.

In the following, we shall use the term of a hyperring, instead of the term of a general hyperring, intending the above definition. A non-empty subset $A$ of $R$ is called a subhyperring of $(R,+, \cdot)$ if $(A,+)$ is a subhypergroup of $(R,+)$ and $A \cdot A \subseteq A$. A subhyperring $A$ of a hyperring $R$ is a left hyperideal of $R$ if $r \cdot a \subseteq A$, for all $r \in R$ and $a \in A$. A right hyperideal of a hyperring $R$ is defined in a similar way. A subhyperring $A$ is called a hyperideal if $A$ is both left and right hyperideal.

The algebraic hypersystem $(R,+, \cdot)$ is a semihyperring if addition and multiplication are both hyperoperations such that $(R,+)$ and $(R, \cdot)$ are semihypergroups and the hyperoperation $\cdot$ is distributive with respect to the hyperoperation + , which means that for all $x, y, z \in R$, we have $x \cdot(y+z)=x \cdot y+x \cdot z$ and $(x+y) \cdot z=x \cdot z+y \cdot z$. A non-empty subset $A$ of $R$ is called a subsemihyperring of $R$ if for all $x, y \in A$, we have $x+y \subseteq A$ and $x \cdot y \subseteq A$. A subsemihyperring $A$ of $R$ is said to be a left hyperideal of $R$ if $r \cdot x \subseteq A$, for all $r \in R$ and $x \in A$. A right hyperideal of a semihyperring $R$ is defined in a similar way. A subsemihyperring $A$ of $R$ which is both a left and a right hyperideal of $R$ is said to be a hyperideal of $R$.

Let $(R,+, \cdot)$ and $\left(T,+^{\prime}, .^{\prime}\right)$ be two (semi)hyperrings. A mapping $\varphi: R \rightarrow T$ is said to be a homomorphism if for all $x, y \in R, \varphi(x+y) \subseteq \varphi(x)+{ }^{\prime} \varphi(y)$ and $\varphi(x \cdot y) \subseteq \varphi(x) \cdot^{\prime} \varphi(y)$. Also $\varphi$ is called a good homomorphism if in the previous conditions (1) and (2), the equality is valid. An isomorphism from $R$ into $T$ is a bijective good homomophism. Let $\sigma$ be an equivalence relation on a (semi)hyperring $(R,+, \cdot)$. We define the following hyperoperations on $R / \sigma$ as follows: $\sigma(x) \oplus \sigma(y)=\{\sigma(z) \mid z \in x+y\}$ and $\sigma(x) \odot \sigma(y)=\{\sigma(z) \mid z \in x \cdot y\}$. Then, the relation $\sigma$ is regular if and only if $(R / \sigma, \oplus, \odot)$ is a (semi)hyperring. Also, $\sigma$ is strongly regular if and only if $(R / \sigma, \oplus, \odot)$ is a (semi)ring.

A partial order is a relation $\leq$ on a non-empty set $R$ which satisfies conditions reflexivity, antisymmetry and transitivity. A relation $\leq$ on a non-empty set $R$ is called a preorder on $R$ if it is reflexive and transitive. By an ordered semigroup, we mean an algebraic structure $(S, \cdot, \leq)$, which satisfies the following properties: (1) $(S, \cdot)$ is a semigroup; (2) $S$ is a partial ordered set by $\leq$; (3) $a \leq b$ implies $a \cdot c \leq b \cdot c$ and $c \cdot a \leq c \cdot b$ for all $a, b, c \in S$. Vandiver [26] gave the first formal definition of 
a semiring in 1934. For an introduction to theory of semirings we refer the readers to [13]. There are different definitions of a semiring. Throughout this paper, a semiring will be defined as follows: A semiring $(R,+, \cdot)$ is an algebraic system together with two binary operations addition and multiplication denoted by + and - respectively, satisfying the following conditions: $(1)(R,+)$ is a (commutative) semigroup; (2) $(R, \cdot)$ is a semigroup; (3) multiplication is distributive from both sides over addition. By zero of a semiring $(R,+, \cdot)$ we mean an element $0 \in R$ such that $x+0=0+x=x$ and $x \cdot 0=0 \cdot x=0$ for all $x \in R$. A semiring with zero and a commutative semigroup $(R,+)$ is called a hemiring. In 2011 , the concept of an ordered semiring was studied by Gan and Jiang [12]. A semiring $(R,+, \cdot)$ is said to be an ordered semiring if there exists a partial order relation $\leq$ on $R$ such that (1) If $a \leq b$ then $a+c \leq b+c$ and $c+a \leq c+b$ for all $a, b, c \in R$; (2) If $a \leq b$ then $a \cdot c \leq b \cdot c$ and $c \cdot a \leq c \cdot b$ for all $a, b, c \in R$.

\section{ORDERED (SEMI)HYPERRINGS}

Let $\sigma$ be an equivalence relation on a (semi)hyperring $(R,+, \cdot)$. If $A$ and $B$ are non-empty subsets of $R$, then $A \bar{\sigma} B$ means that for all $a \in A$, there exists $b \in B$ such that $a \sigma b$ and for all $b^{\prime} \in B$, there exists $a^{\prime} \in A$ such that $a^{\prime} \sigma b^{\prime}$. Also, $A \overline{\bar{\sigma}} B$ means that for all $a \in A$ and for all $b \in B$, we have $a \sigma b$. An equivalence relation $\sigma$ on $R$ is said to be regular if for all $a, b, x \in R$, we have (i) $a \sigma b \Rightarrow$ $(a+x) \bar{\sigma}(b+x)$ and $(x+a) \bar{\sigma}(x+b)$; (ii) $a \sigma b \Rightarrow(a \cdot x) \bar{\sigma}(b \cdot x)$ and $(x \cdot a) \bar{\sigma}(x \cdot b)$. An equivalence relation $\sigma$ on $R$ is said to be strongly regular if for all $a, b, x \in R$, we have (i) $a \sigma b \Rightarrow(a+x) \overline{\bar{\sigma}}(b+x)$ and $(x+a) \overline{\bar{\sigma}}(x+b)$; (ii) $a \sigma b \Rightarrow(a \cdot x) \overline{\bar{\sigma}}(b \cdot x)$ and $(x \cdot a) \overline{\bar{\sigma}}(x \cdot b)$.

Regular and strongly regular relations are important in order to study the quotient structures, as the following theorems show.

Theorem 3.1. Let $(R,+, \cdot)$ be a (semi)hyperring and $\sigma$ be an equivalence relation on $R$. If we define the following hyperoperations on the set of all equivalence classes with respect to $\sigma$, that is, $R / \sigma=\{\sigma(r) \mid r \in R\}$ :

$$
\begin{gathered}
\sigma(x) \oplus \sigma(y)=\{\sigma(z) \mid z \in x+y\}, \\
\sigma(x) \odot \sigma(y)=\{\sigma(z) \mid z \in x \cdot y\},
\end{gathered}
$$

then $\sigma$ is regular if and only if $(R / \sigma, \oplus, \odot)$ is a (semi)hyperring.

Theorem 3.2. An equivalence relation $\sigma$ on a (semi)hyperring $(R,+, \cdot)$ is strongly regular if and only if $(R / \sigma, \oplus, \odot)$ is a (semi)ring.

Let $\varphi$ be a good homomorphism from a (semi)hyperring $(R,+, \cdot)$ to a (semi)hyperring $\left(T,+^{\prime}, \cdot^{\prime}\right)$. Then, the relation $\sigma_{\varphi}=\{(a, b) \in R \times R \mid \varphi(a)=\varphi(b)\}$ on $R$ is called the relation on $R$ induced by $\varphi$.

Lemma 3.3. Let $\varphi$ be a good homomorphism from a (semi)hyperring $(R,+, \cdot)$ to a (semi)hyperring $\left(T,+^{\prime}, \cdot^{\prime}\right)$. Then, $\sigma_{\varphi}$ is a regular equivalence relation on $(R,+, \cdot)$.

Proof. One can see that $\sigma_{\varphi}$ is an equivalence relation on $R$. Let $a, b, x \in R$ be such that $a \sigma_{\varphi} b$. Then $\varphi(a)=\varphi(b)$ and so we have $\varphi(a+x)=\varphi(a)+{ }^{\prime} \varphi(x)=$ $\varphi(b)+{ }^{\prime} \varphi(x)=\varphi(b+x)$. Now, for any $u \in a+x$ there exists $v \in b+x$ such 
that $\varphi(u)=\varphi(v)$, i.e., $u \sigma_{\varphi} v$. Also, for any $v^{\prime} \in b+x$ there exists $u^{\prime} \in a+x$ such that $\varphi\left(u^{\prime}\right)=\varphi\left(v^{\prime}\right)$, i.e., $u^{\prime} \sigma_{\varphi} v^{\prime}$. This implies that $(a+x) \overline{\sigma_{\varphi}}(b+x)$. Similarly, $(x+a) \overline{\sigma_{\varphi}}(x+b),(a \cdot x) \overline{\sigma_{\varphi}}(b \cdot x)$ and $(x \cdot a) \overline{\sigma_{\varphi}}(x \cdot b)$. Hence, $\sigma_{\varphi}$ is a regular equivalence relation on $(R,+, \cdot)$.

One can easily prove the following theorems.

Theorem 3.4. $\left(R / \sigma_{\varphi}, \oplus, \odot\right)$ is a (semi)hyperring.

Theorem 3.5. Let $\varphi$ be a good homomorphism from a (semi)hyperring $(R,+, \cdot)$ to a (semi)hyperring $\left(T,+^{\prime},,^{\prime}\right)$. Then, $R / \sigma_{\varphi} \cong \operatorname{Im} \varphi$.

Theorem 3.6. Let $\sigma$ and $\theta$ be two regular equivalence relation on a (semi)hyperring $(R,+, \cdot)$ such that $\sigma \subseteq \theta$. We define a relation $\theta / \sigma$ on $R / \sigma$ as follows:

$$
(\sigma(a), \sigma(b)) \in \theta / \sigma \Leftrightarrow(a, b) \in \theta .
$$

Then,

(1) $\theta / \sigma$ is a regular equivalence relation on $(R / \sigma, \oplus, \odot)$.

(2) $(R / \sigma) /(\theta / \sigma) \cong R / \theta$.

In the following, we introduce the concept of ordered (semi)hyperring and give some examples that illustrate the significance of this hyperstructure.

Definition 3.7. An algebraic hypersructure $(R,+, \cdot, \leq)$ is called an ordered (semi)hyperring if $(R,+, \cdot)$ is a (semi)hyperring with a partial order $\leq$ such that for all $a, b$, and $c$ in $R$ :

(1) If $a \leq b$, then $a+c \leq b+c$, meaning that for any $x \in a+c$, there exists $y \in b+c$ such that $x \leq y$. The case $c+a \leq c+b$ is defined similarly.

(2) If $a \leq b$ and $c \in R$, then $a \cdot c \leq b \cdot c$, meaning that for any $x \in a \cdot c$, there exists $y \in b \cdot c$ such that $x \leq y$. The case $c \cdot a \leq c \cdot b$ is defined similarly.

Example 3.8. Every (semi)hyperring induces an ordered (semi)hyperring. Indeed: Let $(R,+, \cdot)$ be a (semi)hyperring. Define the order $\leq_{R}$ on $R$ by $\leq_{R}:=\{(x, y) \mid x=$ $y\}$. Then $\left(R,+, \cdot, \leq_{R}\right)$ forms an ordered (semi)hyperring.

Example 3.9. Let $R=\{a, b\}$ be a set with two hyperoperations + and . defined as follows:

\begin{tabular}{|c|cc|}
\hline+ & $a$ & $b$ \\
\hline$a$ & $a$ & $\{a, b\}$ \\
$b$ & $\{a, b\}$ & $\{a, b\}$ \\
\hline
\end{tabular}

\begin{tabular}{|c|cc|}
\hline$\cdot$ & $a$ & $b$ \\
\hline$a$ & $a$ & $\{a, b\}$ \\
$b$ & $a$ & $\{a, b\}$ \\
\hline
\end{tabular}

Then, $(R,+, \cdot)$ is a hyperring. We have $(R,+, \cdot, \leq)$ is an ordered hyperring where the order relation $\leq$ is defined by:

$$
\leq:=\{(a, a),(b, b),(a, b)\} .
$$

The covering relation and the figure of $R$ are given by:

$$
\prec=\{(a, b)\} .
$$




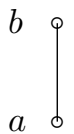

Example 3.10. Let $R=\{a, b\}$ be a set with two hyperoperations + and $\cdot$ defined as follows:

\begin{tabular}{|c|cc|}
\hline+ & $a$ & $b$ \\
\hline$a$ & $a$ & $\{a, b\}$ \\
$b$ & $b$ & $b$
\end{tabular}$\quad \quad$\begin{tabular}{|c|cc|}
$\cdot \cdot$ & $a$ & $b$ \\
\hline$a$ & $a$ & $a$ \\
$b$ & $a$ & $b$ \\
\hline
\end{tabular}

Then, $(R,+, \cdot)$ is a semihyperring. We have $(R,+, \cdot, \leq)$ is an ordered semihyperring where the order relation $\leq$ is defined by:

$$
\leq:=\{(a, a),(b, b),(a, b)\}
$$

The covering relation and the figure of $R$ are given by:

$$
\begin{gathered}
\prec=\{(a, b)\} . \\
b \quad 9 \\
a \quad \\
\end{gathered}
$$

Example 3.11. Let $R=\{0, a, b\}$ be a set with two hyperoperations + and $\cdot$ defined as follows:

\begin{tabular}{|c|ccc|}
\hline+ & 0 & $a$ & $b$ \\
\hline 0 & 0 & $a$ & $b$ \\
$a$ & $a$ & $\{a, b\}$ & $R$ \\
$b$ & $b$ & $R$ & $\{a, b\}$ \\
\hline
\end{tabular}

\begin{tabular}{|c|ccc|}
\hline$\cdot$ & 0 & $a$ & $b$ \\
\hline 0 & 0 & 0 & 0 \\
$a$ & 0 & $R$ & $R$ \\
$b$ & 0 & $R$ & $R$ \\
\hline
\end{tabular}

Then, $(R,+, \cdot)$ is a hyperring. We have $(R,+, \cdot, \leq)$ is an ordered hyperring where the order relation $\leq$ is defined by:

$$
\leq:=\{(0,0),(a, a),(b, b),(0, a),(0, b),(a, b)\}
$$

The covering relation and the figure of $R$ are given by:

$$
\prec=\{(0, a),(a, b)\} .
$$




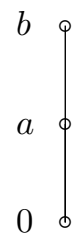

Example 3.12. Let $R=\{a, b, c, d\}$ be a set with two hyperoperations + and . defined as follows:

\begin{tabular}{|c|cccc|}
\hline+ & $a$ & $b$ & $c$ & $d$ \\
\hline$a$ & $a$ & $\{a, b\}$ & $\{c, d\}$ & $\{c, d\}$ \\
$b$ & $\{a, b\}$ & $\{a, b\}$ & $\{c, d\}$ & $\{c, d\}$ \\
$c$ & $\{c, d\}$ & $\{c, d\}$ & $\{a, b\}$ & $\{a, b\}$ \\
$d$ & $\{c, d\}$ & $\{c, d\}$ & $\{a, b\}$ & $\{a, b\}$ \\
\hline
\end{tabular}

\begin{tabular}{|c|cccc|}
\hline$\cdot$ & $a$ & $b$ & $c$ & $d$ \\
\hline$a$ & $\{a, b\}$ & $\{a, b\}$ & $\{a, b\}$ & $\{a, b\}$ \\
$b$ & $\{a, b\}$ & $\{a, b\}$ & $\{a, b\}$ & $\{a, b\}$ \\
$c$ & $\{a, b\}$ & $\{a, b\}$ & $\{c, d\}$ & $\{c, d\}$ \\
$d$ & $\{a, b\}$ & $\{a, b\}$ & $\{c, d\}$ & $\{c, d\}$ \\
\hline
\end{tabular}

Then, $(R,+, \cdot)$ is a hyperring [8]. We have $(R,+, \cdot, \leq)$ is an ordered hyperring where the order relation $\leq$ is defined by:

$$
\leq:=\{(a, a),(b, b),(c, c),(d, d),(a, b),(c, d)\} .
$$

The covering relation and the figure of $R$ are given by:

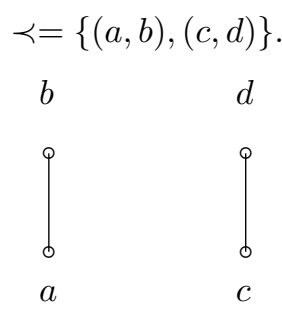

Example 3.13. Let $R=\{0, a, b, c, d\}$ be a set with the following hyperoperations:

\begin{tabular}{|c|ccccc|}
\hline+ & 0 & $a$ & $b$ & $c$ & $d$ \\
\hline 0 & 0 & $a$ & $b$ & $c$ & $d$ \\
$a$ & $a$ & $\{0, a\}$ & $b$ & $c$ & $d$ \\
$b$ & $b$ & $b$ & $\{0, a\}$ & $d$ & $c$ \\
$c$ & $c$ & $c$ & $d$ & $\{0, a\}$ & $b$ \\
$d$ & $d$ & $d$ & $c$ & $b$ & $\{0, a\}$ \\
\hline
\end{tabular}

and

\begin{tabular}{|c|ccccc|}
\hline$\cdot$ & 0 & $a$ & $b$ & $c$ & $d$ \\
\hline 0 & 0 & 0 & 0 & 0 & 0 \\
$a$ & 0 & $\{0, a\}$ & $\{0, a\}$ & $\{0, a\}$ & $\{0, a\}$ \\
$b$ & 0 & $\{0, a\}$ & $\{0, a\}$ & $\{0, a\}$ & $\{0, a\}$ \\
$c$ & 0 & $\{0, a\}$ & $\{0, a\}$ & $\{0, a\}$ & $\{0, a\}$ \\
$d$ & 0 & $\{0, a\}$ & $\{0, a\}$ & $\{0, a\}$ & $\{0, a\}$ \\
\hline
\end{tabular}


Then, $(R,+, \cdot)$ is a hyperring [31]. We have $(R,+, \cdot, \leq)$ is an ordered hyperring where the order relation $\leq$ is defined by:

$$
\leq:=\{(0,0),(0, a),(a, a),(b, b),(c, c),(d, d)\} .
$$

The covering relation and the figure of $R$ are given by:

$$
\prec=\{(0, a)\} .
$$

$$
\begin{array}{cccc}
\circ & & & \\
& \circ & \circ & \circ \\
0 & b & c & d
\end{array}
$$

Example 3.14. Let $R=\{a, b, c, d, e\}$ be a set with the following hyperoperations:

\begin{tabular}{|c|ccccc|}
\hline+ & $a$ & $b$ & $c$ & $d$ & $e$ \\
\hline$a$ & $a$ & $\{b, c\}$ & $\{b, c\}$ & $d$ & $e$ \\
$b$ & $\{b, c\}$ & $d$ & $d$ & $e$ & $a$ \\
$c$ & $\{b, c\}$ & $d$ & $d$ & $e$ & $a$ \\
$d$ & $d$ & $e$ & $e$ & $a$ & $\{b, c\}$ \\
$e$ & $e$ & $a$ & $a$ & $\{b, c\}$ & $d$ \\
\hline
\end{tabular}

and

\begin{tabular}{|c|ccccc|}
\hline$\cdot$ & $a$ & $b$ & $c$ & $d$ & $e$ \\
\hline$a$ & $a$ & $a$ & $a$ & $a$ & $a$ \\
$b$ & $a$ & $\{b, c\}$ & $\{b, c\}$ & $d$ & $e$ \\
$c$ & $a$ & $\{b, c\}$ & $\{b, c\}$ & $d$ & $e$ \\
$d$ & $a$ & $d$ & $d$ & $a$ & $d$ \\
$e$ & $a$ & $e$ & $e$ & $d$ & $\{b, c\}$ \\
\hline
\end{tabular}

Then, $(R,+, \cdot)$ is a commutative hyperring [8]. We have $(R,+, \cdot, \leq)$ is an ordered hyperring where the order relation $\leq$ is defined by:

$$
\leq:=\{(a, a),(b, b),(c, c),(d, d),(e, e),(b, c)\} .
$$

The covering relation and the figure of $R$ are given by:

$$
\begin{aligned}
& \prec=\{(b, c)\} . \\
& \text { c } \\
& \begin{array}{llll}
\circ & 0 & & \\
a & 0 & d & e \\
& b & &
\end{array}
\end{aligned}
$$


Example 3.15. Let $R=\{a, b, c, d, e, f, g\}$ be a set with the hyperaddition + defined as follows:

\begin{tabular}{|c|ccccccc|}
\hline+ & $a$ & $b$ & $c$ & $d$ & $e$ & $f$ & $g$ \\
\hline$a$ & $\{a, b\}$ & $\{a, b\}$ & $c$ & $d$ & $e$ & $f$ & $g$ \\
$b$ & $\{a, b\}$ & $\{a, b\}$ & $c$ & $d$ & $e$ & $f$ & $g$ \\
$c$ & $c$ & $c$ & $\{a, b\}$ & $f$ & $g$ & $d$ & $e$ \\
$d$ & $d$ & $d$ & $g$ & $\{a, b\}$ & $f$ & $e$ & $c$ \\
$e$ & $e$ & $e$ & $f$ & $g$ & $\{a, b\}$ & $c$ & $d$ \\
$f$ & $f$ & $f$ & $e$ & $c$ & $e$ & $g$ & $\{a, b\}$ \\
$g$ & $g$ & $g$ & $d$ & $e$ & $c$ & $\{a, b\}$ & $f$ \\
\hline
\end{tabular}

we define a hyperoperation - as follows:

$$
x \cdot y=\{a, b\}, \forall x, y \in R .
$$

Then, $(R,+, \cdot)$ is a hyperring $[21]$. We have $(R,+, \cdot, \leq)$ is an ordered hyperring where the order relation $\leq$ is defined by:

$$
\leq \quad:=\{(a, a),(b, b),(c, c),(d, d),(e, e),(f, f),(g, g),(a, b)\} .
$$

The covering relation and the figure of $R$ are given by:

$$
\prec=\{(a, b)\} \text {. }
$$

$b$

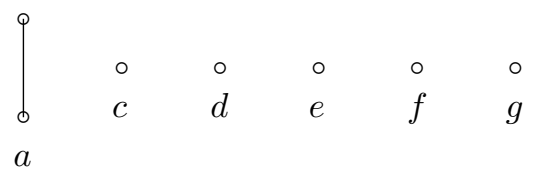

Proposition 3.16. Let $\left(R,+, \cdot, \leq_{R}\right)$ and $\left(T,+^{\prime}, \cdot^{\prime}, \leq_{T}\right)$ be two ordered (semi)hyperrings. Then, $R \times T$ is an ordered (semi)hyperring where for all $\left(s_{1}, t_{1}\right)$ and $\left(s_{2}, t_{2}\right)$ in $R \times T$ we define
(1) $\left(s_{1}, t_{1}\right) \uplus\left(s_{2}, t_{2}\right)=\left\{(x, y) \mid x \in s_{1}+s_{2}, y \in t_{1}+{ }^{\prime} t_{2}\right\}$,
(2) $\left(s_{1}, t_{1}\right) \otimes\left(s_{2}, t_{2}\right)=\left\{(x, y) \mid x \in s_{1} \cdot s_{2}, y \in t_{1} \cdot{ }^{\prime} t_{2}\right\}$,
(3) $\left(s_{1}, t_{1}\right) \preceq\left(s_{2}, t_{2}\right)$ if and only if $s_{1} \leq_{R} s_{2}$ and $t_{1} \leq_{T} t_{2}$.

Proof. The proof is straightforward.

In the following theorem, for all $x \in R$, the class of $x$ is denoted by $\bar{x}$ and defined by $\bar{x}=\{y \mid x \sigma y\}$. The quotient of $R$ with respect to $\sigma$ is denoted by $R / \sigma$ and defined by $R / \sigma=\{\bar{x} \mid x \in R\}$.

Theorem 3.17. Let $(R,+, \cdot, \leq)$ be a partially preordered (semi)hyperring and $\sigma$ be a strongly regular relation on $R$. Then, $(R / \sigma, \oplus, \odot, \preceq)$ is a partially preordered (semi)ring with respect to the following hyperoperations on the quotient set $R / \sigma$ as follows:

$$
\begin{gathered}
\bar{a} \oplus \bar{b}=\{\bar{c} \mid c \in a+b\}, \\
\bar{a} \odot \bar{b}=\{\bar{c} \mid c \in a \cdot b\},
\end{gathered}
$$


where for all $\bar{a}, \bar{b} \in R / \sigma$ the preorder relation $\preceq$ is defined by:

$$
\bar{a} \preceq \bar{b} \Leftrightarrow \forall a_{1} \in \bar{a} \exists b_{1} \in \bar{b} \text { such that } a_{1} \leq b_{1} .
$$

Proof. $\sigma$ is a strongly regular relation on $R$. Hence, by Theorem $3.2,(R / \sigma, \oplus, \odot)$ is a (semi)ring. First, we show that the binary relation $\preceq$ is a partial preorder relation on $R / \sigma$. Since $a \leq a$, so $\bar{a} \preceq \bar{a}$ for every $\bar{a} \in R / \sigma$. Thus $\preceq$ is reflexive. Now, let $\bar{a} \preceq \bar{b}$ and $\bar{b} \preceq \bar{a}$. Take any $a_{1} \in \bar{a}$; then there exists $b_{1} \in \bar{b}$ such that $a_{1} \leq b_{1}$. For this $b_{1} \in \bar{b}$ there exists $c_{1} \in \bar{c}$ such that $b_{1} \leq c_{1}$. Hence for every $a_{1} \in \bar{a}$ there exists some $c_{1} \in \bar{c}$ such that $a_{1} \leq c_{1}$. This implies that $\bar{a} \preceq \bar{c}$. So we have shown that $\preceq$ is transitive.

The $\oplus, \odot$ hyperoperations are compatible with the $\preceq$ preorder. To see this, suppose that $\bar{a}, \bar{b}, \bar{x} \in R / \sigma, \bar{a} \preceq \bar{b}$. If $\bar{u}=\bar{x} \oplus \bar{a}$, then for every $u_{1} \in \bar{u}$ there exist $x_{1} \in \bar{x}$ and $a_{1} \in \bar{a}$ such that $u_{1} \in x_{1}+a_{1}$. Since $a_{1} \in \bar{a} \preceq \bar{b}$ there exists $b_{1} \in \bar{b}$ such that $a_{1} \leq b_{1}$. Hence $x_{1}+a_{1} \leq x_{1}+b_{1}$. Thus there exists $v_{1} \in x_{1}+b_{1}$ such that $u_{1} \leq v_{1}$. Therefore, $\bar{u}=\overline{u_{1}} \preceq \bar{v}_{1}=\bar{x} \oplus \bar{b}$. If $\bar{s}=\bar{x} \odot \bar{a}$, then for every $s_{1} \in \bar{s}$ there exist $x_{1} \in \bar{x}$ and $a_{1} \in \bar{a}$ such that $s_{1} \in x_{1} \cdot a_{1}$. Since $a_{1} \in \bar{a} \preceq \bar{b}$ there exists $b_{1} \in \bar{b}$ such that $a_{1} \leq b_{1}$. Hence $x_{1} \cdot a_{1} \leq x_{1} \cdot b_{1}$. Thus there exists $t_{1} \in x_{1} \cdot b_{1}$ such that $s_{1} \leq t_{1}$. Therefore, $\bar{s}=\overline{s_{1}} \preceq \overline{t_{1}}=\bar{x} \odot \bar{b}$. Therefore, $(R / \sigma, \oplus, \odot, \preceq)$ is a partially preordered (semi)ring.

Definition 3.18. Let $(R,+, \cdot, \leq)$ be an ordered hyperring. A non-empty subset I of $R$ is called a hyperideal of $R$ if it satisfies the following conditions:

(1) $(I,+)$ is a subhypergroup of $(R,+)$;

(2) $(I \cdot R) \cup(R \cdot I) \subseteq I$;

(3) When $x \in I$ and $y \in R$ such that $y \leq x$, imply that $y \in I$.

Example 3.19. In Example 3.14, $\{a, d\}$ is a hyperideal of $R$.

In the theory of hyperrings, fundamental relations make a connection between hyperrings and ordinary rings. The relation $\gamma^{*}$ is the smallest strongly regular relation and it is called the fundamental relation on the hyperring $(R,+, \cdot)$. In order to see which elements are in the equivalence relation $\gamma^{*}$, we denote the set of all finite hypersums of finite hyperproducts of elements of $(R,+, \cdot)$ by $\mathcal{U}$ and we have $x \gamma y \Leftrightarrow \exists u \in \mathcal{U}$ such that $\{x, y\} \subset u$.

Definition 3.20. $[29,30]$ Let $(R,+, \cdot)$ be a hyperring. We define the relation $\gamma$ as follows: $x \gamma y \Leftrightarrow \exists n \in \mathbb{N}, \exists k_{i} \in \mathbb{N}, \exists\left(x_{i 1}, \cdots, x_{i k_{i}}\right) \in R^{k_{i}}, 1 \leq i \leq n$, such that $\{x, y\} \subseteq \sum_{i=1}^{n}\left(\prod_{j=1}^{k_{i}} x_{i j}\right)$. The relation $\gamma$ is reflexive and symmetric. Let $\gamma^{*}$ be the transitive closure of $\gamma$.

In $[29,30]$ it was proved that

Theorem 3.21. Let $(R,+, \cdot)$ be a hyperring. Then,

(1) $\gamma^{*}$ is a strongly regular relation both on $(R,+)$ and $(R, \cdot)$.

(2) The quotient $R / \gamma^{*}$ is a ring.

(3) The relation $\gamma^{*}$ is the smallest equivalence relation on $R$ such that the quotient $R / \gamma^{*}$ is a ring. $R / \gamma^{*}$ is called the fundamental ring. 


\section{Pseudoorder in ordered (SEMi)hyperringS}

In [18], Kehayopulu and Tsingelis gave the example of an ordered semigroup $(S, \cdot, \leq)$ and a congruence $\sigma$ on $S$ such that the relation $\preceq$ on $S / \sigma$, denoted by

$\preceq:=\{(\sigma(a), \sigma(b)) \in S / \sigma \times S / \sigma \mid \exists x \in \sigma(a), \exists y \in \sigma(b)$ such that $(x, y) \in \leq\}$,

is not an order relation on $S / \sigma$, in general. Let $(R,+, \cdot)$ be a semihyperring and $\sigma$ an equivalence relation on $R$. We define the following hyperoperations on the quotient set $R / \sigma: \sigma(x) \oplus \sigma(y)=\{\sigma(z) \mid z \in x+y\}$ and $\sigma(x) \odot \sigma(y)=\{\sigma(z) \mid z \in x \cdot y\}$. It is well known that $\sigma$ is a strongly regular relation if and only if $(R / \sigma, \oplus, \odot)$ is a semiring. Now, the following question is natural: If $(R,+, \cdot, \leq)$ is an ordered semihyperring and $\sigma$ is a strongly regular relation on $R$, then is the set $R / \sigma$ an ordered semiring? A probable order on $R / \sigma$ could be the relation $\preceq$ on $R / \sigma$ defined by means of the order $\leq$ on $R$, that is,

$$
\preceq:=\{(\sigma(a), \sigma(b)) \in R / \sigma \times R / \sigma \mid \exists x \in \sigma(a), \exists y \in \sigma(b) \text { such that }(x, y) \in \leq\} .
$$

But this relation is not an order, in general. It is enough we consider the following example.

Example 4.1. Let $R=\{a, b, c, d, e\}$ be a set with two hyperoperations + and . defined as follows:

\begin{tabular}{|c|ccccc|}
\hline+ & $a$ & $b$ & $c$ & $d$ & $e$ \\
\hline$a$ & $\{b, c\}$ & $\{b, d\}$ & $\{b, d\}$ & $\{b, d\}$ & $\{b, d\}$ \\
$b$ & $\{b, d\}$ & $\{b, d\}$ & $\{b, d\}$ & $\{b, d\}$ & $\{b, d\}$ \\
$c$ & $\{b, d\}$ & $\{b, d\}$ & $\{b, d\}$ & $\{b, d\}$ & $\{b, d\}$ \\
$d$ & $\{b, d\}$ & $\{b, d\}$ & $\{b, d\}$ & $\{b, d\}$ & $\{b, d\}$ \\
$e$ & $\{b, d\}$ & $\{b, d\}$ & $\{b, d\}$ & $\{b, d\}$ & $\{b, d\}$ \\
\hline
\end{tabular}

and

\begin{tabular}{|c|ccccc|}
\hline$\cdot$ & $a$ & $b$ & $c$ & $d$ & $e$ \\
\hline$a$ & $\{b, d\}$ & $\{b, d\}$ & $\{b, d\}$ & $\{b, d\}$ & $\{b, d\}$ \\
$b$ & $\{b, d\}$ & $\{b, d\}$ & $\{b, d\}$ & $\{b, d\}$ & $\{b, d\}$ \\
$c$ & $\{b, d\}$ & $\{b, d\}$ & $\{b, d\}$ & $\{b, d\}$ & $\{b, d\}$ \\
$d$ & $\{b, d\}$ & $\{b, d\}$ & $\{b, d\}$ & $\{b, d\}$ & $\{b, d\}$ \\
$e$ & $\{b, d\}$ & $\{b, d\}$ & $\{b, d\}$ & $\{b, d\}$ & $\{b, d\}$ \\
\hline
\end{tabular}

Then, $(R,+, \cdot)$ is a semihyperring. We have $(R,+, \cdot, \leq)$ is an ordered semihyperring where the order relation $\leq$ is defined by:

$$
\leq:=\{(a, a),(b, b),(b, e),(c, c),(c, d),(d, d),(e, e)\} .
$$

The covering relation and the figure of $R$ are given by:

$$
\prec=\{(b, e),(c, d)\} .
$$




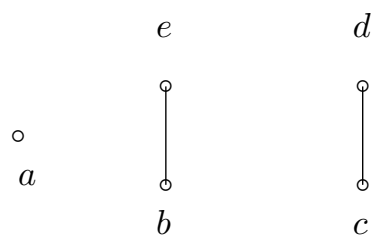

Let $\sigma$ be a strongly regular relation on $R$ defined as follows:

$$
\sigma:=\{(a, a),(b, b),(b, d),(c, c),(c, e),(d, b),(d, d),(e, c),(e, e)\} .
$$

Let $\preceq$ be an order on $R / \sigma$ defined by means of the order $\leq$ on $R$, that is,

$$
\preceq:=\{(\sigma(a), \sigma(b)) \mid \exists x \in \sigma(a), \exists y \in \sigma(b) \text { such that }(x, y) \in \leq\} .
$$

We have $\sigma(b)=\{b, d\}$ and $\sigma(c)=\{c, e\}$. Also,

$$
\begin{gathered}
\sigma(b) \preceq \sigma(c) \text { since } b \in \sigma(b), e \in \sigma(c),(b, e) \in \leq, \\
\sigma(c) \preceq \sigma(b) \text { since } c \in \sigma(c), d \in \sigma(b),(c, d) \in \leq .
\end{gathered}
$$

Since $\sigma(b) \neq \sigma(c)$, it follows that $\preceq$ is not an order relation on $R / \sigma$.

The following question arises:

Question. Let $(R,+, \cdot, \leq)$ be an ordered (semi)hyperring. Is there a strongly regular relation $\sigma$ on $R$ for which $R / \sigma$ is an ordered (semi)ring?

Our main aim in the following is reply to the above question. This leads us to the concept of pseudoorders of ordered (semi)hyperrings.

Davvaz et al. [10], investigated the relationship between ordered semihypergroups and ordered semigroups by using pseudoorders. In case of ordered semigroups, pseudoorders play the role of congruences of semigroups. The concept of pseudoorder on an ordered semigroup $(S, \cdot, \leq)$ was introduced and studied by Kehayopula and Tsingelis $[18,19]$. Now, we extend this notion for ordered (semi)hyperrings. We continue this section with the following definition.

Definition 4.2. Let $(R,+, \cdot, \leq)$ be an ordered (semi)hyperring. A relation $\sigma$ on $R$ is called pseudoorder if for all $a, b, c \in R$, we have
(1) $\leq \subseteq \sigma$,
(2) $a \sigma b$ and $b \sigma c$ imply $a \sigma c$,
(3) $a \sigma b$ implies $a+c \overline{\bar{\sigma}} b+c$ and $c+a \overline{\bar{\sigma}} c+b$,

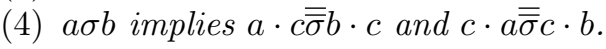

Lemma 4.3. Let $\left\{\sigma_{i} \mid i \in \Omega\right\}$ be a set of pseudoorders on an ordered (semi)hyperring $(R,+, \cdot, \leq)$. Then, $\sigma=\bigcap_{i \in \Omega} \sigma_{i}$ is a pseudoorder on $(R,+, \cdot, \leq)$.

Proof. Obviously, $\leq \subseteq \sigma$ and $\sigma$ is transitive. Now, let $a \sigma b$ and $c \in R$. Then $a \sigma_{i} b$ for all $i \in \Omega$. Since each $\sigma_{i}$ is a pseudoorder on $R$, by conditions (3) and (4) of Definition 4.2 , we conclude that

$$
\begin{aligned}
& a+c \overline{\overline{\sigma_{i}}} b+c, \\
& c+a \overline{\overline{\sigma_{i}}} c+b,
\end{aligned}
$$




$$
\begin{aligned}
& a \cdot c \overline{\overline{\sigma_{i}}} b \cdot c, \\
& c \cdot a \overline{\overline{\sigma_{i}}} c \cdot b .
\end{aligned}
$$

Hence, for every $u \in a+c$ and $v \in b+c$, we have $u \sigma_{i} v$ for all $i \in \Omega$. So, we have $u \sigma v$. This implies that $a+c \overline{\bar{\sigma}} b+c$. Similarly, we obtain $c+a \overline{\bar{\sigma}} c+b, a \cdot c \overline{\bar{\sigma}} b \cdot c$ and $c \cdot a \overline{\bar{\sigma}} c \cdot b$. Thus, $\sigma$ is a pseudoorder on $R$.

Definition 4.4. Let $\Theta=\left\{\theta_{i} \mid i \in \Omega\right\}$ be a family of pseudoorders on an ordered (semi)hyperring $(R,+, \cdot, \leq)$. We say that $\Theta$ separates the elements of $R$ if for each $x, y \in R,(x, y) \notin \leq$, there exists $\theta_{i} \in \Theta$ such that $(x, y) \notin \theta_{i}$.

Lemma 4.5. Let $\Theta=\left\{\theta_{i} \mid i \in \Omega\right\}$ be a family of pseudoorders on an ordered (semi)hyperring $(R,+, \cdot, \leq)$. If $\Theta$ separates the elements of $R$, then $\leq=\bigcap_{i \in \Omega} \theta_{i}$. Conversely, if $\bigcap_{i \in \Omega} \theta_{i} \subseteq \leq$, then $\Theta$ separates the elements of $R$.

Proof. The proof is similar to the proof of Lemma 3 in [18].

In the following, by using the notion of pseudoorder, we obtain an ordered semiring from an ordered semihyperring.

Theorem 4.6. Let $(R,+, \cdot, \leq)$ be an ordered semihyperring and $\sigma$ a pseudoorder on $R$. Then, there exists a strongly regular equivalence relation $\sigma^{*}=\{(a, b) \in$ $R \times R \mid a \sigma b$ and $b \sigma a\}$ on $R$ such that $\left(R / \sigma^{*}, \oplus, \odot, \preceq\right)$ is an ordered semiring, where $\preceq:=\left\{\left(\sigma^{*}(x), \sigma^{*}(y)\right) \in R / \sigma^{*} \times R / \sigma^{*} \mid \exists a \in \sigma^{*}(x), \exists b \in \sigma^{*}(y)\right.$ such that $\left.(a, b) \in \sigma\right\}$.

Proof. Suppose that $\sigma^{*}$ is the relation on $R$ defined as follows:

$$
\sigma^{*}=\{(a, b) \in R \times R \mid a \sigma b \text { and } b \sigma a\} .
$$

First, we show that $\sigma^{*}$ is a strongly regular relation on $(R,+)$ and $(R, \cdot)$. Since $(a, a) \in \leq$ and $\leq \subseteq \sigma$, we have $a \sigma a$. So, $a \sigma^{*} a$. If $(a, b) \in \sigma^{*}$, then $a \sigma b$ and $b \sigma a$. Hence, $(b, a) \in \sigma^{*}$. If $(a, b) \in \sigma^{*}$ and $(b, c) \in \sigma^{*}$, then $a \sigma b, b \sigma a, b \sigma c$ and $c \sigma b$. Hence, $a \sigma c$ and $c \sigma a$, which imply that $(a, c) \in \sigma^{*}$. Thus $\sigma^{*}$ is an equivalence relation. Now, let $a \sigma^{*} b$ and $c \in R$. Then $a \sigma b$ and $b \sigma a$. Since $\sigma$ is a pseudoorder on $R$, by conditions (3) and (4) of Definition 4.2 , we conclude that

$$
\begin{array}{cl}
a+c \overline{\bar{\sigma}} b+c, & c+a \overline{\bar{\sigma}} c+b, \\
b+c \overline{\bar{\sigma}} a+c, & c+b \overline{\bar{\sigma}} c+a, \\
a \cdot c \overline{\bar{\sigma}} b \cdot c, & c \cdot a \overline{\bar{\sigma}} c \cdot b, \\
b \cdot c \overline{\bar{\sigma}} a \cdot c, & c \cdot b \overline{\bar{\sigma}} c \cdot a .
\end{array}
$$

Hence, for every $x \in a+c$ and $y \in b+c$, we have $x \sigma y$ and $y \sigma x$ which imply that $x \sigma^{*} y$. So, $a+c \overline{\overline{\sigma^{*}}} b+c$. Similarly, we have $c+a \overline{\overline{\sigma^{*}}} c+b$. Thus, $\sigma^{*}$ is a strongly regular relation on $(R,+)$. Clearly, $\sigma^{*}$ is a strongly regular relation on $(R, \cdot)$. Hence, by Theorem $3.2, R / \sigma^{*}$ with the following operations is a semiring:

$$
\begin{aligned}
& \sigma^{*}(x) \oplus \sigma^{*}(y)=\sigma^{*}(z), \text { for every } z \in x+y \\
& \sigma^{*}(x) \odot \sigma^{*}(y)=\sigma^{*}(w), \text { for every } w \in x \cdot y
\end{aligned}
$$


Now, we define a relation $\preceq$ on $R / \sigma^{*}$ as follows:

$\preceq:=\left\{\left(\sigma^{*}(x), \sigma^{*}(y)\right) \in R / \sigma^{*} \times R / \sigma^{*} \mid \exists a \in \sigma^{*}(x), \exists b \in \sigma^{*}(y)\right.$ such that $\left.(a, b) \in \sigma\right\}$.

We show that

$$
\sigma^{*}(x) \preceq \sigma^{*}(y) \Leftrightarrow x \sigma y .
$$

Let $\sigma^{*}(x) \preceq \sigma^{*}(y)$. We show that for every $a \in \sigma^{*}(x)$ and $b \in \sigma^{*}(y)$, a $\sigma b$. Since $\sigma^{*}(x) \preceq \sigma^{*}(y)$, there exist $x^{\prime} \in \sigma^{*}(x)$ and $y^{\prime} \in \sigma^{*}(y)$ such that $x^{\prime} \sigma y^{\prime}$. Since $a \in \sigma^{*}(x)$ and $x^{\prime} \in \sigma^{*}(x)$, we obtain $a \sigma^{*} x^{\prime}$, and so $a \sigma x^{\prime}$ and $x^{\prime} \sigma a$. Since $b \in \sigma^{*}(y)$ and $y^{\prime} \in \sigma^{*}(y)$, we obtain $b \sigma^{*} y^{\prime}$, and so $b \sigma y^{\prime}$ and $y^{\prime} \sigma b$. Now, we have $a \sigma x^{\prime}, x^{\prime} \sigma y^{\prime}$ and $y^{\prime} \sigma b$, which imply that $a \sigma b$. Since $x \in \sigma^{*}(x)$ and $y \in \sigma^{*}(y)$, we conclude that $x \sigma y$. Conversely, let $x \sigma y$. Since $x \in \sigma^{*}(x)$ and $y \in \sigma^{*}(y)$, we obtain $\sigma^{*}(x) \preceq \sigma^{*}(y)$.

Finally, we prove that $\left(R / \sigma^{*}, \oplus, \odot, \preceq\right)$ is an ordered semiring. Suppose that $\sigma^{*}(x) \in R / \sigma^{*}$, where $x \in R$. Then, $(x, x) \in \leq \subseteq \sigma$. Hence, $\sigma^{*}(x) \preceq \sigma^{*}(x)$. Let $\sigma^{*}(x) \preceq \sigma^{*}(y)$ and $\sigma^{*}(y) \preceq \sigma^{*}(x)$. Then, $x \sigma y$ and $y \sigma x$. Thus, $x \sigma^{*} y$, which means that $\sigma^{*}(x)=\sigma^{*}(y)$. Now, let $\sigma^{*}(x) \preceq \sigma^{*}(y)$ and $\sigma^{*}(y) \preceq \sigma^{*}(z)$. Then, $x \sigma y$ and $y \sigma z$. So, we have $x \sigma z$. This implies that $\sigma^{*}(x) \preceq \sigma^{*}(z)$. Hence $\preceq$ is an order on $R / \sigma^{*}$.

Now, let $\sigma^{*}(x) \preceq \sigma^{*}(y)$ and $\sigma^{*}(z) \in R / \sigma^{*}$. Then $x \sigma y$ and $z \in R$. By conditions (3) and (4) of Definition 4.2, we have $x+z \overline{\bar{\sigma}} y+z, z+x \overline{\bar{\sigma}} z+y, x \cdot z \overline{\bar{\sigma}} y \cdot z$ and $z \cdot x \overline{\bar{\sigma}} z \cdot y$. So, for all $a \in x+z$ and $b \in y+z$, we have $a \sigma b$. This implies that $\sigma^{*}(a) \preceq \sigma^{*}(b)$. Hence, $\sigma^{*}(x) \oplus \sigma^{*}(z) \preceq \sigma^{*}(y) \oplus \sigma^{*}(z)$. Similarly, we get $\sigma^{*}(z) \oplus \sigma^{*}(x) \preceq \sigma^{*}(z) \oplus \sigma^{*}(y)$. Also, for all $a \in x \cdot z$ and $b \in y \cdot z$, we have $a \sigma b$. This implies that $\sigma^{*}(a) \preceq \sigma^{*}(b)$. Hence, $\sigma^{*}(x) \odot \sigma^{*}(z) \preceq \sigma^{*}(y) \odot \sigma^{*}(z)$. Similarly, we get $\sigma^{*}(z) \odot \sigma^{*}(x) \preceq \sigma^{*}(z) \odot \sigma^{*}(y)$. Hence, the theorem is proved.

Analogous to the proof of Theorem 4.6, we have the following result.

Theorem 4.7. Let $(R,+, \cdot, \leq)$ be an ordered hyperring and $\sigma$ be a pseudoorder on $R$. Then, there exists a strongly regular relation $\sigma^{*}$ on $R$ such that $R / \sigma^{*}$ is an ordered ring.

Example 4.8. Let $R=\{a, b, c, d, e\}$ be a set with two hyperoperations + and . defined as follows:

\begin{tabular}{|c|ccccc|}
\hline+ & $a$ & $b$ & $c$ & $d$ & $e$ \\
\hline$a$ & $\{b, c\}$ & $\{b, d\}$ & $\{b, d\}$ & $\{b, d\}$ & $e$ \\
$b$ & $\{b, d\}$ & $\{b, d\}$ & $\{b, d\}$ & $\{b, d\}$ & $e$ \\
$c$ & $\{b, d\}$ & $\{b, d\}$ & $\{b, d\}$ & $\{b, d\}$ & $e$ \\
$d$ & $\{b, d\}$ & $\{b, d\}$ & $\{b, d\}$ & $\{b, d\}$ & $e$ \\
$e$ & $\{b, d\}$ & $\{b, d\}$ & $\{b, d\}$ & $\{b, d\}$ & $e$ \\
\hline
\end{tabular}

and

\begin{tabular}{|c|ccccc|}
\hline$\cdot$ & $a$ & $b$ & $c$ & $d$ & $e$ \\
\hline$a$ & $\{b, d\}$ & $\{b, d\}$ & $\{b, d\}$ & $\{b, d\}$ & $\{b, d\}$ \\
$b$ & $\{b, d\}$ & $\{b, d\}$ & $\{b, d\}$ & $\{b, d\}$ & $\{b, d\}$ \\
$c$ & $\{b, d\}$ & $\{b, d\}$ & $\{b, d\}$ & $\{b, d\}$ & $\{b, d\}$ \\
$d$ & $\{b, d\}$ & $\{b, d\}$ & $\{b, d\}$ & $\{b, d\}$ & $\{b, d\}$ \\
$e$ & $\{b, d\}$ & $\{b, d\}$ & $\{b, d\}$ & $\{b, d\}$ & $\{b, d\}$ \\
\hline
\end{tabular}


Then, $(R,+, \cdot)$ is a semihyperring [22]. We consider the ordered semihyperring $(R,+, \cdot, \leq)$, where the order $\leq$ is defined by:

$$
\leq:=\{(a, a),(b, b),(c, c),(d, d),(e, e),(c, b),(d, b),(e, b)\} .
$$

The covering relation and the figure of $R$ are given by:

$$
\prec=\{(c, b),(d, b),(e, b)\} .
$$

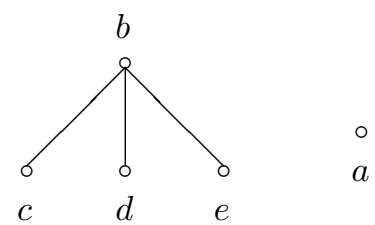

Let $\sigma$ be pseudoorder on $R$ define as follows:

$$
\begin{aligned}
\sigma= & \{(a, a),(b, b),(c, c),(d, d),(e, e),(b, c),(c, b), \\
& (b, d),(d, b),(b, e),(e, b),(c, d),(d, c),(c, e), \\
& (e, c),(d, e),(e, d),(a, b),(a, c),(a, d),(a, e)\} .
\end{aligned}
$$

Then, by the definition of $\rho^{*}$, we get

$$
\begin{aligned}
\sigma^{*}= & \{(a, a),(b, b),(c, c),(d, d),(e, e),(b, c),(c, b), \\
& (b, d),(d, b),(b, e),(e, b),(c, d),(d, c),(c, e), \\
& (e, c),(d, e),(e, d)\} .
\end{aligned}
$$

Hence, $R / \sigma^{*}=\left\{u_{1}, u_{2}\right\}$, where $u_{1}=\{a\}$ and $u_{2}=\{b, c, d, e\}$. Now, $\left(R / \sigma^{*}, \oplus, \odot, \preceq\right.$ ) is an ordered semiring, where $\oplus$ and $\odot$ are defined in the following tables:

\begin{tabular}{|c|ll|}
\hline$\oplus$ & $u_{1}$ & $u_{2}$ \\
\hline$u_{1}$ & $u_{2}$ & $u_{2}$ \\
$u_{2}$ & $u_{2}$ & $u_{2}$ \\
\hline
\end{tabular}

\begin{tabular}{|c|cc|}
\hline$\odot$ & $u_{1}$ & $u_{2}$ \\
\hline$u_{1}$ & $u_{2}$ & $u_{2}$ \\
$u_{2}$ & $u_{2}$ & $u_{2}$ \\
\hline
\end{tabular}

and $\preceq=\left\{\left(u_{1}, u_{1}\right),\left(u_{1}, u_{2}\right),\left(u_{2}, u_{2}\right)\right\}$.

Example 4.9. Suppose that $R=\{a, b, c, d\}$. We consider the ordered semihyperring $(R,+, \cdot, \leq)$, where the operations + and $\cdot$ are defined by the following tables:

\begin{tabular}{|c|llll|}
\hline+ & $a$ & $b$ & $c$ & $d$ \\
\hline$a$ & $a$ & $b$ & $c$ & $d$ \\
$b$ & $b$ & $b$ & $c$ & $d$ \\
$c$ & $c$ & $c$ & $c$ & $d$ \\
$d$ & $d$ & $d$ & $d$ & $d$ \\
\hline
\end{tabular}

\begin{tabular}{|c|cccc|}
\hline$\cdot$ & $a$ & $b$ & $c$ & $d$ \\
\hline$a$ & $a$ & $a$ & $a$ & $a$ \\
$b$ & $a$ & $b$ & $b$ & $b$ \\
$c$ & $a$ & $b$ & $b$ & $b$ \\
$d$ & $a$ & $b$ & $b$ & $b$ \\
\hline
\end{tabular}

and the order $\leq$ is defined by:

$$
\begin{aligned}
\leq \quad:= & \{(a, a),(b, b),(c, c),(d, d),(a, b),(a, c),(a, d), \\
& (b, c),(b, d),(c, d)\} .
\end{aligned}
$$

The covering relation and the figure of $R$ are given by:

$$
\prec=\{(a, b),(b, c),(c, d)\} .
$$




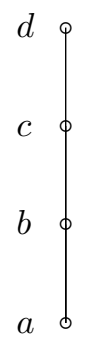

Let $\sigma$ be pseudoorder on $R$ define as follows:

$$
\begin{aligned}
\sigma= & \{(a, a),(b, b),(c, c),(d, d),(a, b),(b, a),(a, c), \\
& (c, a),(b, c),(c, b),(a, d),(b, d),(c, d)\} .
\end{aligned}
$$

Then, by the definition of $\sigma^{*}$, we get

$$
\begin{aligned}
\sigma^{*}= & \{(a, a),(b, b),(c, c),(d, d),(a, b),(b, a),(a, c), \\
& (c, a),(b, c),(c, b)\} .
\end{aligned}
$$

Hence, $R / \sigma^{*}=\left\{u_{1}, u_{2}\right\}$, where $u_{1}=\{a, b, c\}$ and $u_{2}=\{d\}$. Now, $\left(R / \sigma^{*}, \oplus, \odot, \preceq\right)$ is an ordered semiring, where $\oplus$ and $\odot$ are defined in the following tables:

\begin{tabular}{|l|ll|}
\hline$\oplus$ & $u_{1}$ & $u_{2}$ \\
\hline$u_{1}$ & $u_{1}$ & $u_{2}$ \\
$u_{2}$ & $u_{2}$ & $u_{2}$ \\
\hline
\end{tabular}

\begin{tabular}{|c|cc|}
\hline$\odot$ & $u_{1}$ & $u_{2}$ \\
\hline$u_{1}$ & $u_{1}$ & $u_{1}$ \\
$u_{2}$ & $u_{1}$ & $u_{1}$ \\
\hline
\end{tabular}

and $\preceq=\left\{\left(u_{1}, u_{1}\right),\left(u_{1}, u_{2}\right),\left(u_{2}, u_{2}\right)\right\}$.

Definition 4.10. Let $\left(R,+, \cdot, \leq_{R}\right)$ and $\left(T,+^{\prime},{ }^{\prime}, \leq_{T}\right)$ be two ordered (semi)hyperrings. The map $\varphi: R \rightarrow T$ is called a homomorphism if for all $x, y \in R$, the following conditions hold:

(1) $\varphi(x+y) \subseteq \varphi(x)+{ }^{\prime} \varphi(y)$,

(2) $\varphi(x \cdot y) \subseteq \varphi(x) \cdot{ }^{\prime} \varphi(y)$,

(3) $\varphi$ is isotone, that is, $x \leq_{R} y$ implies $\varphi(x) \leq_{T} \varphi(y)$.

Also, $\varphi$ is called a good homomorphism if in the previous conditions (1) and (2), the equality is valid. An isomorphism from $R$ into $T$ is a bijective good homomorphism. Note that if $\left(R,+, \cdot, \leq_{R}\right)$ and $\left(T,{ }^{\prime},{ }^{\prime}, \leq_{T}\right)$ are two ordered (semi)rings, then the notions of homomorphism and good homomorphisms coincide.

Theorem 4.11. Let $\left(R,+, \cdot, \leq_{R}\right)$ and $\left(T,{ }^{\prime},{ }^{\prime}, \leq_{T}\right)$ be two ordered (semi)rings and $\varphi: R \rightarrow T$ a homomorphism. The relation $\sigma$ on $R$ defined by $\sigma:=\left\{(x, y) \mid \varphi(x) \leq_{T}\right.$ $\varphi(y)\}$ is a pseudoorder on $R$.

Proof. Suppose that $(x, y) \in \leq_{R}$. Since $\varphi$ is a homomorphism, it follows that $\varphi(x) \leq_{T} \varphi(y)$. This means that $(x, y) \in \sigma$. So, we have $\leq_{R} \subseteq \sigma$. Let $(x, y) \in \sigma$ and $(y, z) \in \sigma$. Then, we have $\varphi(x) \leq_{T} \varphi(y)$ and $\varphi(y) \leq_{T} \varphi(z)$. Since $\leq_{T}$ is transitive, we have $\varphi(x) \leq_{T} \varphi(z)$. This implies that $(x, z) \in \sigma$. 
Now, let $(x, y) \in \sigma$ and $z \in R$. Then $\varphi(x) \leq_{T} \varphi(y)$. Since $\varphi$ is a homomorphism and $T$ an ordered (semi)ring, we have

$$
\begin{gathered}
\varphi(x+z)=\varphi(x)+^{\prime} \varphi(z) \leq_{T} \varphi(y)+^{\prime} \varphi(z)=\varphi(y+z), \\
\varphi(x \cdot z)=\varphi(x) \cdot^{\prime} \varphi(z) \leq_{T} \varphi(y) \cdot^{\prime} \varphi(z)=\varphi(y \cdot z) .
\end{gathered}
$$

So, $(x+z, y+z) \in \sigma$ and $(x \cdot z, y \cdot z) \in \sigma$. Similarly, we have $(z+x, z+y) \in \sigma$ and $(z \cdot x, z \cdot y) \in \sigma$. Therefore, $\sigma$ is a pseudoorder on $R$.

Corollary 4.12. $\sigma_{\varphi}=\sigma^{*}$.

Proof. We have

$$
\begin{aligned}
(x, y) \in \sigma_{\varphi} & \Leftrightarrow \varphi(x)=\varphi(y) \\
& \Leftrightarrow \varphi(x) \leq_{T} \varphi(y) \text { and } \varphi(y) \leq_{T} \varphi(x) \\
& \Leftrightarrow(x, y) \in \sigma \text { and }(y, x) \in \sigma \\
& \Leftrightarrow(x, y) \in \sigma^{*} .
\end{aligned}
$$

Corollary 4.13. Let $\left(R,+, \cdot, \leq_{R}\right)$ and $\left(T,+^{\prime}, \cdot^{\prime}, \leq_{T}\right)$ be two ordered (semi)rings and $\varphi: R \rightarrow T$ be a homomorphism. Then, $R / \operatorname{ker} \varphi \cong \operatorname{Im} \varphi$.

Let $\left(R,+, \cdot, \leq_{R}\right)$ be an ordered (semi)hyperring, $\sigma, \theta$ be pseudoorders on $R$ such that $\sigma \subseteq \theta$. We define a relation $\theta / \sigma$ on $R / \sigma^{*}$ as follows:

$\theta / \sigma:=\left\{\left(\sigma^{*}(a), \sigma^{*}(b)\right) \in R / \sigma^{*} \times R / \sigma^{*} \mid \exists x \in \sigma^{*}(a), \exists y \in \sigma^{*}(b)\right.$ such that $\left.(x, y) \in \theta\right\}$.

Then, we can see that

$$
\left(\sigma^{*}(a), \sigma^{*}(b)\right) \in \theta / \sigma \Leftrightarrow(a, b) \in \theta .
$$

In the following theorem, we denote by $\preceq_{\sigma}$ the relation $\preceq$ on $R / \sigma^{*}$ defined in Theorem 4.6.

Theorem 4.14. Let $\left(R,+, \cdot, \leq_{R}\right)$ be an ordered (semi)hyperring, $\sigma, \theta$ be pseudoorders on $R$ such that $\sigma \subseteq \theta$. Then,

(1) $\theta / \sigma$ is a pseudoorder on $R / \sigma^{*}$.

(2) $\left(R / \sigma^{*}\right) /(\theta / \sigma)^{*} \cong R / \theta^{*}$.

Proof. (1) If $\left(\sigma^{*}(a), \sigma^{*}(b)\right) \in \preceq_{\sigma}$, then $(a, b) \in \sigma$. So, $(a, b) \in \theta$ which implies that $\left(\sigma^{*}(a), \sigma^{*}(b)\right) \in \theta / \sigma$. Thus, $\preceq_{\sigma} \subseteq \theta / \sigma$. Let $\left(\sigma^{*}(a), \sigma^{*}(b)\right) \in \theta / \sigma$ and $\left(\sigma^{*}(b), \sigma^{*}(c)\right) \in$ $\theta / \sigma$. Then $(a, b) \in \theta$ and $(b, c) \in \theta$. Hence, $(a, c) \in \theta$ and so $\left(\sigma^{*}(a), \sigma^{*}(c)\right) \in \theta / \sigma$. Now, let $\left(\sigma^{*}(a), \sigma^{*}(b)\right) \in \theta / \sigma$ and $\sigma^{*}(c) \in R / \sigma^{*}$. Then, $(a, b) \in \theta$. Since $\theta$ is a pseudoorder on $R$, we obtain $a+c \overline{\bar{\theta}} b+c, c+a \overline{\bar{\theta}} c+b, a \cdot c \overline{\bar{\theta}} b \cdot c$ and $c \cdot a \overline{\bar{\theta}} c \cdot b$. Hence, for all $x \in a+c$ and for all $y \in b+c$, we have $(x, y) \in \theta$. This implies that $\left(\sigma^{*}(x), \sigma^{*}(y)\right) \in \theta / \sigma$. Also, for all $x^{\prime} \in a \cdot c$ and for all $y^{\prime} \in b \cdot c$, we have $\left(x^{\prime}, y^{\prime}\right) \in \theta$. This implies that $\left(\sigma^{*}\left(x^{\prime}\right), \sigma^{*}\left(y^{\prime}\right)\right) \in \theta / \sigma$. Since $\sigma^{*}$ is a strongly regular relation on $R$, $\sigma^{*}(x)=\sigma^{*}(a) \oplus \sigma^{*}(c), \sigma^{*}(y)=\sigma^{*}(b) \oplus \sigma^{*}(c), \sigma^{*}\left(x^{\prime}\right)=\sigma^{*}(a) \odot \sigma^{*}(c)$ and $\sigma^{*}\left(y^{\prime}\right)=$ $\sigma^{*}(b) \odot \sigma^{*}(c)$. So, we obtain $\left(\sigma^{*}(a) \oplus \sigma^{*}(c), \sigma^{*}(b) \oplus \sigma^{*}(c)\right) \in \theta / \sigma$ and $\left(\sigma^{*}(a) \odot\right.$ $\left.\sigma^{*}(c), \sigma^{*}(b) \odot \sigma^{*}(c)\right) \in \theta / \sigma$. Similarly, we obtain $\left(\sigma^{*}(c) \oplus \sigma^{*}(a), \sigma^{*}(c) \oplus \sigma^{*}(b)\right) \in \theta / \sigma$ 
and $\left(\sigma^{*}(c) \odot \sigma^{*}(a), \sigma^{*}(c) \odot \sigma^{*}(b)\right) \in \theta / \sigma$. Therefore, $\theta / \sigma$ is a pseudoorder on $R / \sigma^{*}$.

(2) We define the map $\psi: R / \sigma^{*} \rightarrow R / \theta^{*}$ by $\psi\left(\sigma^{*}(a)\right)=\theta^{*}(a)$. If $\sigma^{*}(a)=$ $\sigma^{*}(b)$, then $(a, b) \in \sigma^{*}$. Hence, by the definition of $\sigma^{*},(a, b) \in \sigma \subseteq \theta$ and $(b, a) \in$ $\sigma \subseteq \theta$. This implies that $(a, b) \in \theta^{*}$ and so $\theta^{*}(a)=\theta^{*}(b)$. Thus, $\psi$ is well defined. For all $\sigma^{*}(x), \sigma^{*}(y) \in R / \sigma^{*}$, we have

$$
\begin{aligned}
& \sigma^{*}(x) \oplus \sigma^{*}(y)=\sigma^{*}(z), \text { for all } z \in x+y, \\
& \theta^{*}(x) \uplus \theta^{*}(y)=\theta^{*}(z), \text { for all } z \in x+y, \\
& \sigma^{*}(x) \odot \sigma^{*}(y)=\sigma^{*}(z), \text { for all } z \in x \cdot y, \\
& \theta^{*}(x) \otimes \theta^{*}(y)=\theta^{*}(z), \text { for all } z \in x \cdot y .
\end{aligned}
$$

Thus,

and

$$
\begin{aligned}
\psi\left(\sigma^{*}(x) \oplus \sigma^{*}(y)\right) & =\psi\left(\sigma^{*}(z)\right), \text { for all } z \in x+y \\
& =\theta^{*}(z), \text { for all } z \in x+y \\
& =\theta^{*}(x) \uplus \theta^{*}(y) \\
& =\psi\left(\sigma^{*}(x)\right) \uplus \psi\left(\sigma^{*}(y)\right),
\end{aligned}
$$

$$
\begin{aligned}
\psi\left(\sigma^{*}(x) \odot \sigma^{*}(y)\right) & =\psi\left(\sigma^{*}(z)\right), \text { for all } z \in x \cdot y \\
& =\theta^{*}(z), \text { for all } z \in x \cdot y \\
& =\theta^{*}(x) \otimes \theta^{*}(y) \\
& =\psi\left(\sigma^{*}(x)\right) \otimes \psi\left(\sigma^{*}(y)\right),
\end{aligned}
$$

and if $\sigma^{*}(x) \preceq_{\sigma} \sigma^{*}(y)$, then $(x, y) \in \sigma$. So, $(x, y) \in \theta$ and this implies that $\theta^{*}(x) \preceq_{\theta} \theta^{*}(y)$. Therefore, $\psi$ is a homomorphism. It is easy to see that $\psi$ is onto, since

$$
\operatorname{Im} \psi=\left\{\psi\left(\sigma^{*}(x)\right) \mid x \in R\right\}=\left\{\theta^{*}(x) \mid x \in R\right\}=R / \theta^{*} .
$$

So, by Corollary 4.13, we obtain

$$
\left(R / \sigma^{*}\right) / k e r \psi \cong \operatorname{Im} \psi=R / \theta^{*} .
$$

Suppose that

$$
k:=\left\{\left(\sigma^{*}(x), \sigma^{*}(y)\right) \mid \psi\left(\sigma^{*}(x)\right) \preceq{ }_{\theta} \psi\left(\sigma^{*}(y)\right)\right\}
$$

Then,

$$
\begin{aligned}
\left(\sigma^{*}(x), \sigma^{*}(y)\right) \in k & \Leftrightarrow \psi\left(\sigma^{*}(x)\right) \preceq \preceq_{\theta} \psi\left(\sigma^{*}(y)\right) \\
& \Leftrightarrow \theta^{*}(x) \preceq \theta^{*}(y) \\
& \Leftrightarrow(x, y) \in \theta \\
& \Leftrightarrow\left(\sigma^{*}(x), \sigma^{*}(y)\right) \in \theta / \sigma .
\end{aligned}
$$

Hence, $k=\theta / \sigma$ and by Corollary 4.12 , we have $k^{*}=(\theta / \sigma)^{*}=k e r \psi$.

\section{REFERENCES}

[1] Asokkumar, A. and Velrajan, M., "Hyperring of matrices over a regular hyperring", Ital. J. Pure Appl. Math., 23 (2008), 113-120.

[2] Bakhshi, M. and Borzooei, R.A., "Ordered polygroups", Ratio Math., 24 (2013), 31-40.

[3] Changphas, T. and Davvaz, B., "Properties of hyperideals in ordered semihypergroups", Ital. J. Pure Appl. Math., 33 (2014), 425-432.

[4] Corsini, P., Prolegomena of Hypergroup Theory, Aviani Editore, Tricesimo, 1993. 
[5] Corsini, P. and Leoreanu, V., Applications of Hyperstructure Theory, Adv. Math., Kluwer Academic Publishers, Dordrecht, 2003.

[6] Chvalina, J., "Commutative hypergroups in the sence of Marty and ordered sets", Proceedings of the Summer School in General Algebra and Ordered Sets, Olomouck, (1994), 19-30.

[7] Chvalina, J. and Moucka, J., "Hypergroups determined by orderings with regular endomorphism monoids", Ital. J. Pure Appl. Math., 16 (2004), 227-242.

[8] Davvaz, B. and Leoreanu-Fotea, V., Hyperring Theory and Applications, International Academic Press, Palm Harbor, USA, 2007.

[9] Davvaz, B. and Vougiouklis, T., "Commutative rings obtained from hyperrings $\left(H_{v}\right.$-rings) with $\alpha^{*}$-relations", Comm. Algebra, 35 (2007), 3307-3320.

[10] Davvaz, B., Corsini, P. and Changphas, T., "Relationship between ordered semihypergroups and ordered semigroups by using pseudoorder", European J. Combinatorics, 44 (2015), 208217.

[11] De Salvo, M., "Hyperrings and hyperfields", Annales Scientifiques de Universite de Clermont-Ferrand II, 22 (1984), 89-107.

[12] Gan, A.P. and Jiang, Y.L., "On ordered ideals in ordered semirings", J. Math. Res. Exposition, 31 (2011), 989-996.

[13] Golan, J.S., Semirings and Their Applications, Kluwer Acad. Publ., Dordrecht, 1999.

[14] Gu, Z. and Tang, X., "Ordered regular equivalence relations on ordered semihypergroups", J. Algebra, 450 (2016), 384-397.

[15] Heidari, D. and Davvaz, B., "On ordered hyperstructures", Politehn. Univ. Bucharest Sci. Bull. Ser. A Appl. Math. Phys., 73(2) (2011), 85-96.

[16] Hort, D.A., "A construction of hypergroups from ordered structures and their morphisms", J. Discrete Math. Sci. Cryptogr. 6 (2003), 139-150.

[17] Hoskova, S., "Upper order hypergroups as a reflective subcategory of subquasiorder hypergroups", Ital. J. Pure Appl. Math., 20 (2006), 215-222.

[18] Kehayopulu, N. and Tsingelis, M., "On subdirectly irreducible ordered semigroups", Semigroup Forum, 50 (1995), 161-177.

[19] Kehayopulu, N. and Tsingelis, M., "Pseudoorder in ordered semigroups", Semigroup Forum, 50 (1995), 389-392.

[20] Marty, F., "Sur une generalization de la notion de groupe", giem Congres Math. Scandinaves, Stockholm, (1934), 45-49.

[21] Mirvakili, S. and Davvaz, B., "Strongly transitive geometric spaces: applications to hyperrings", Rev. Un. Mat. Argentina, 53 (2012), 43-53.

[22] Mirvakili, S. and Davvaz, B., "Relationship between rings and hyperrings by using the notion of fundamental relations", Comm. Algebra, 41 (2013), 70-82.

[23] Rahnamai Barghi, A., "A class of hyperrings", J. Discrete Math. Sci. Cryptogr., 6 (2003), 227-233.

[24] Spartalis, S., "A class of hyperrings", Rivista Mat. Pura Appl., 4 (1989), 55-64.

[25] Tang, J., Davvaz, B. and Luo, Y.F., "Hyperfilters and fuzzy hyperfilters of ordered semihypergroups", J. Intell. Fuzzy Systems, 29 (2015), 75-84.

[26] Vandiver, H.S., "Note on a simple type of algebra in which the cancellation law of addition does not hold", Bull. Amer. Math. Soc., 40 (1934), 914-920.

[27] Velrajan, M. and Asokkumar, A., "Note on isomorphism theorems of hyperrings", Int. J. Math. and Math. Sci., (2010), 12 pages.

[28] Vougiouklis, T., "On some representations of hypergroups", Ann. Sci. Univ. ClermontFerrand II Math., 26 (1990), 21-29.

[29] Vougiouklis, T., Hyperstructures and Their Representations, Hadronic Press Inc., Florida, 1994.

[30] Vougiouklis, T., "The fundamental relation in hyperrings. The general hyperfields", Proc. Fourth Int. Congress on Algebraic Hyperstructures and Applications (AHA 1990), World Sci. Publ., (1991), 203-211. 
[31] Yin, Y. and Huang, X., "Fuzzy roughness in hyperrings based on a complete residuated lattice", Int. J. Fuzzy Syst., 13 (2011), 185-194. 